\title{
Pulsed-dose-rate peri-operative brachytherapy as an interstitial boost in organ-sparing treatment of breast cancer
}

\author{
Krystyna Serkies, MD, PhD', Janusz Jaśkiewicz, MD, PhD², Rafał Dziadziuszko, MD, PhD', Jacek Jassem, MD, PhD' \\ 'Department of Oncology and Radiotherapy, Medical University of Gdańsk, Gdansk, ${ }^{2}$ Department of Plastic Surgery, Medical University \\ of Gdańsk, Gdańsk, Poland
}

\begin{abstract}
Purpose: To evaluate peri-operative multicatheter interstitial pulsed-dose-rate brachytherapy (PDR-BT) with an intra-operative catheter placement to boost the tumor excision site in breast cancer patients treated conservatively.

Material and methods: Between May 2002 and October 2008, 96 consecutive T1-3N0-2M0 breast cancer patients underwent breast-conserving therapy (BCT) including peri-operative PDR-BT boost, followed by whole breast external beam radiotherapy (WBRT). The BT dose of $15 \mathrm{~Gy}$ (1 Gy/pulse/h) was given on the following day after surgery.

Results: No increased bleeding or delayed wound healing related to the implants were observed. The only side effects included one case of temporary peri-operative breast infection and 3 cases of fat necrosis, both early and late. In 11 patients $(11.4 \%)$, subsequent WBRT was omitted owing to the final pathology findings. These included eight patients who underwent mastectomy due to multiple adverse prognostic pathological features, one case of lobular carcinoma in situ, and two cases with no malignant tumor. With a median follow-up of 12 years (range: 7-14 years), among 85 patients who completed BCT, there was one ipsilateral breast tumor and one locoregional nodal recurrence. Six patients developed distant metastases and one was diagnosed with angiosarcoma within irradiated breast. The actuarial 5- and 10-year disease free survival was 90\% (95\% CI: 84-96\%) and 87\% (95\% CI: 80-94\%), respectively, for the patients with invasive breast cancer, and 91\% (95\% CI: 84-97\%) and 89\% (95\% CI: 82-96\%), respectively, for patients who completed BCT. Good cosmetic outcome by self-assessment was achieved in 58 out of 64 (91\%) evaluable patients.

Conclusions: Peri-operative PDR-BT boost with intra-operative tube placement followed by EBRT is feasible and devoid of considerable toxicity, and provides excellent long-term local control. However, this strategy necessitates careful patient selection and histological confirmation of primary diagnosis.
\end{abstract}

J Contemp Brachytherapy 2016; 8, 6: 492-496 DOI: $10.5114 /$ jcb.2016.64512

Key words: brachytherapy, breast cancer, breast conserving therapy, PDR.

\section{Purpose}

Breast conserving therapy (BCT) is currently considered as the standard management of early breast cancer. Reduced risk of local recurrence in patients administered a boost dose of 16 Gy to the tumor bed in addition to 50 Gy delivered to the whole breast, was confirmed in a large randomized trial $[1,2]$. This effect was particularly apparent in a subset of patients younger than 50 years. Locoregional recurrence is associated with distant metastases and increased mortality. Boost options include interstitial brachytherapy (BT), electron or photon therapy, all following or preceding whole breast radiotherapy (WBRT) using external beam $[1,3,4,5,6,7]$. In all above approaches, the extent of boost tumor volume may be incorrect if the tumor bed is determined using clinical parameters (e.g., palpation, pre-operative mammography, scar position, operative and pathology reports, or surgical clips placed at the excision site boundaries) $[8,9,10]$. The direct visualization of the operative site during surgery allows for decreasing the risk of "geographical miss" in determining the target volume.

Pulsed-dose-rate (PDR) treatment is a BT modality combining the physical advantages of high-dose-rate (HDR) technology (isodose optimization, radiation safety) with radiobiological advantages of conventional lowdose-rate (LDR) BT. Despite its favorable radiobiological features, PDR-BT has rarely been used as a component of BCT. Here, we present our experience with this method used as an interstitial boost.

\section{Material and methods}

Study group included 96 consecutive patients with microscopically confirmed early breast cancer, 17 of whom,
Address for correspondence: Krystyna Serkies, MD, PhD, Department of Oncology and Radiotherapy, Medical University of Gdańsk, 7 Dębinki St., 80-211 Gdańsk, Poland, phone/ fax: +48 5834922 70, $\bowtie$ e-mail: kserkies@gumed.edu.pl
Received: 12.09 .2016

Accepted: 17.11 .2016

Published: 30.12 .2016 
with initial T1-3 or N1-2 tumor, received induction chemotherapy. All patients underwent BCT between May 2002 and October 2008, including peri-operative PDR-BT boost following intra-operative BT tube placement to the primary tumor excision site (Table 1) and were technically suitable for BT. Breast carcinoma was primarily diagnosed using excisional or tru-cut biopsy in 59 women $(61.5 \%)$ and by fine needle aspiration biopsy in the remaining 37 patients (38.5\%). Flexible tubes were implanted during breast conserving surgery (BCS) including primary tumor excision or re-excision, with immediate tumor cavity reconstruction using surrounding breast tissue in all but one case. Axillary lymph node management included either sentinel node biopsy or nodal dissection. The BT implant covered the tumor excision site and the 1-2 cm margin of normal breast whenever possible. The number of tubes ranged between 4 and 17 (median: 9). In most of patients, in order to guide needles, the standardized templates for a triangular array with a space of 10-14 mm were used. On the following day, $2 \mathrm{D}$ radiographic verifications of tube placement with the skin markers were taken, digitized, and entered into a BT planning system (PLATO, version 13.7 or 14.1, Nucletron, an Elekta company, Elekta AB, Stockholm, Sweden). The target volume was calculated based on the actual position of catheters. The skin dose was reduced by keeping a distance of at least $10 \mathrm{~mm}$ from the first dwell position of the stepping source. The dosimetry was calculated using volume optimization technique. A total dose of 15 Gy (1 Gy per pulse repeated every hour) was delivered. Brachytherapy was followed by external beam radiotherapy (EBRT) to the entire breast after the final histology of the excised tissue had been obtained.

Follow-up data including cosmetic outcome assessed by a patient were obtained through personal contact or phone interview. Classification of side effects was performed using the Common Terminology Criteria for Adverse Events version 4.0, and late toxicity was reported if it occurred at least 6 months after BT.

Statistical analysis was performed with SPSS software (version 13.0, IBM, USA). Time to event endpoints was estimated using Kaplan-Meier method from the date of brachytherapy to the date of any local, regional or distant relapse, or death from breast cancer, whichever occurred first, or to the date of last visit in case of no events.

\section{Results}

On the average, tube implantation prolonged time of surgery by no more than 20 minutes. In three cases with deep located tumor, to construct the deep plane close to or upon the pectoralis minor muscle, tubes were implanted before excision cavity closing. Detailed BT implant and some dosimetric parameters were reported elsewhere [11]. The average volume for the prescribed dose $\left(\mathrm{V}_{100}\right)$ was $34.1 \mathrm{cc}$ (range: $10.8-95.6 \mathrm{cc}$ ), and the median $\mathrm{V}_{100}$ for 93 patients with invasive tumor was $31.2 \mathrm{cc}$. At the time of the analysis, data regarding another dose-volume parameters were available in 36 patients ( $38.7 \%$ of all patients with invasive breast cancer). In this subgroup, the median volume of tissue receiving $150 \%\left(\mathrm{~V}_{150}\right)$ of the prescribed dose was $10.7 \mathrm{cc}$ (range:
Table 1. Patient and treatment characteristics

$(n=96)$

Variable

$n(\%)$

Age (years)

\begin{tabular}{lc}
\hline Range & $27-72$ \\
\hline Median & 51 \\
\hline$\leq 40$ & $9(9.4)$ \\
\hline $41-50$ & $34(35.4)$
\end{tabular}

TNM stage at presentation

T1 N0-2 78 (81)

T2 N0-2 16 (17)

T3 NO 2(2)

Prior chemotherapy

$17(17.7)$

Histology

Invasive ductal 71 (74)

Invasive lobular

$11(11)$

Other

$14(15)$

Final margin status $(n=94)$

\begin{tabular}{lc}
\hline Negative & $80(85.1)$ \\
\hline Positive $^{a, b}$ & $5(5.3)$ \\
\hline Close $^{a}(\leq 5 \mathrm{~mm})$ & $9(9.6)$
\end{tabular}

Surgery

Primary 68 (70.8)

Re-excision after excisional biopsy 28 (29.1)

Sentinel node biopsy 16 (16.6)

Axillary excision and sampling $\quad 80(83.3)$

Brachytherapy implant

\begin{tabular}{lc}
\hline One-plane & $7(7)$ \\
\hline Two-plane & $81(84)$ \\
\hline Three-plane & $8(8)$
\end{tabular}

Number of tubes

\begin{tabular}{lc}
\hline Range & $4-17$ \\
\hline Median & 9
\end{tabular}

Volume at the prescribed isodose $\left(\mathrm{V}_{100}\right)$ (cc)

\begin{tabular}{lc}
\hline Range & $10.8-95.5$ \\
\hline Mean & 34.1
\end{tabular}

Whole breast radiotherapy $(n=85)$

50 Gy/25 fractions $67(78.8)$

42.5 Gy/17 fractions or 40.05 Gy/15 fractions 18 (21.2)

Adjuvant therapy $(n=93)$

\begin{tabular}{ll}
\hline Chemotherapy & $30(32.2)$ \\
\hline Hormonal therapy & $58(62.4)$
\end{tabular}

$a_{-}$invasive and/or in situ ductal carcinoma present at an linked margin, ${ }^{b}-$ in cluding three patients with invasive ductal carcinoma; an adequate margins in two of them was unachievable due to tumor adjunction to the chest wall, the third one underwent subsequent mastectomy, $V_{100}$ - the percent volume of the post-implant receiving $100 \%$ of the prescribed dose, $\mathrm{cc}-\mathrm{cm}^{3}$ 
2.87-33.44 cc), and the median dose homogeneity index [DHI], defined as 1- $\left(\mathrm{V}_{150} / \mathrm{V}_{100}\right)$, was 0.72 (range: 0.53-0.78). In 91 patients, BT has started the day after the implant placement, and in five patients BT was delayed by 1-2 days. Subsequent WBRT was abandoned in 11 patients (11.4\%). These included eight cases with multiple adverse prognostic pathological factors diagnosed postoperatively, implying the superiority of mastectomy, one with the final diagnosis of lobular carcinoma in situ, and two with no malignant tumor; all originally were diagnosed with fine needle aspiration as carcinoma. The remaining 85 patients received WBRT, including one with massive axillary lymph node involvement, in whom breast irradiation was preceded by chemotherapy. Except this case, the break between BT and WBRT ranged from 8 to 31 days (median: 12 days).

No intense bleeding during surgery or at tube removal was observed and neither there were wound healing problems or significant skin reactions related to the implant. One patient with re-excision experienced temporary peri-operative breast infection requiring antibiotic administration. No routine analgesics during therapy or at tube removal were administered. One patient with large-sized breast underwent subsequent surgical intervention due to grade 3 fat necrosis. In this case, WBRT was delayed up to 31 days after BT. Late grade 3 fat necrosis occurred in another two patients. Overall, four patients $(4.3 \%)$ experienced serious side effects.

After median follow-up of 12 years (range: 7-14), one case of "true" local recurrence and one regional nodal recurrence were observed (1.2\% each). Four patients (4.7\%) developed contralateral breast carcinoma (of another histology in two patients or another histological grade in two patients). Six years after BCT, one patient presented with angiosarcoma within the irradiated breast outside of the primary breast cancer. Another seven patients (8.2\%) developed second cancer including lung, ovarian, colon, skin cancer, and lymphoma. Apart from a case with angiosarcoma and a case with disseminated ovarian cancer, six relapsed breast cancer patients died due to cancer dissemination.

The actuarial 5- and 10-year disease-free survival was 90\% (95\% CI: 84-96\%), and 87\% (95\% CI: 80-94\%), respectively, for the whole cohort of 93 invasive breast cancer patients, and 91\% (95\% CI: 84-97\%) and 89\% (95\% CI: 82$96 \%)$, respectively, for patients who completed BCT.

In 58 out of $64(91 \%)$ assessable patients, a good or excellent self-assessed cosmetic result was obtained. The remaining four patients scored cosmetic effect as fair and two patients as poor. One patient with fair cosmetic effect had no tumor excision site reconstruction during BCS, and another one, due to centrally located tumor, required the nipple-areolar complex excision.

\section{Discussion}

Intra-operative irradiation using electron beams, photon beams, or BT tube implantation provides a high precision boost, thus minimizing the risk of a "geographical miss" $[12,13,14,15]$. We demonstrated the feasibility, good tolerance, and efficacy of the peri-operative BT using PDR. The only severe toxicity included three cases of fat necrosis. Interstitial PDR-BT boost seems to be particularly suitable component of $\mathrm{BCT}$, owing to its hypothetical favorable cosmetic outcomes. High local control and satisfactory cosmesis with PDR boost following the whole breast EBRT in breast cancer was reported by other authors [4,5].

The rationale for a BT boost is the delivery of a high dose to the tumor bed with reduced exposure of the skin, lung, and subcutaneous tissue. The important advantages of intra-operative implant include reduced risk of "geographical miss", shortening the treatment time, and avoidance of another anesthesia. An apparent limitation of this approach is the lack of the full pathology assessment, especially regarding the margin status at the time of BT.

In this series, 28 patients (29\%) underwent a re-excision, and in the breast cancer group the tumor resection margins were tumor-free in $85 \%$ of patients. Among cases with positive or close margin, none developed local recurrence. Positive pathologic margin has been considered a major risk factor for ipsilateral breast tumor recurrence (IBTR), although the use of higher boost dose in these cases is debatable [16,17]. A substantial risk of residual disease was reported for breast cancer patients with $<2 \mathrm{~mm}$ margin of excision [18]. In the large retrospective study of 8485 early breast cancer patients with $5 \%$ IBTR incidence at 10 -years, the invasive carcinoma margin status did not influence the risk of local relapse [19]. In this cohort, $9 \%$ of patients underwent a re-excision, and IBTR-free interval was longer for patients who received a RT boost or systemic therapy. A multidisciplinary consensus from 2014 defined an adequate breast cancer margin as no ink on tumor [20]. The role of surgical resection margins after breast-conserving surgery is summarized in recently published Senonetwork recommendations [21]. This document proposes standards for investigating resection margins and recommends in patients with positive margins re-excision or mastectomy, or increasing the boost dose during radiotherapy. In case of negative margins, boost administration and its dose depend on the estimated risk of local recurrence, which is linked to demographic and pathological tumor features as well as the width of surgical margin.

In this series, diagnosis was established using excisional or tru-cut biopsy in the majority of patients; nevertheless, in $11.4 \%$ of patients subsequent WBRT was omitted due to the postoperative pathology findings. All these patients were primarily diagnosed by fine needle aspiration cytology used in the first period of this study. Thus, obtaining the definite diagnosis by a tru-cut biopsy seems to be essential in all patients considered for this strategy.

The optimal total and boost doses as well as BT dose rate, have not yet been determined in BCT. In this series, 15 Gy boost dose and $1 \mathrm{~Gy} /$ pulse/h, followed by $50 \mathrm{~Gy}$ or 40.05 Gy and 42.5 Gy (2 Gy or 2.67 and 2.5 Gy daily dose, respectively) to the whole breast were used. Others administered 20-25 Gy as the PDR boost following breast 50 Gy EBRT [4,5]. Harms et al. [5] tailored the PDR boost dose according to pathologic tumor characteristics. These authors found that the $25 \mathrm{~Gy}$ boost dose is associated with a significantly higher rate of late toxicity compared to $20 \mathrm{~Gy}$. Others reported poorer cosmetic outcome in patients boosted with dose rates above $1 \mathrm{~Gy} / \mathrm{h}$ [22]. Until recently, in patients managed with BCT, the standard WBRT dose was $50 \mathrm{~Gy}$. Recently, mildly hypofractionated 
schedules of radiotherapy are commonly used. Indications and modalities of radiotherapy boost in hypofractionated schedules have not been fully determined. The boost dose was used in $43-75 \%$ of patients enrolled in three out of four randomized trials of hypofractionated radiotherapy as a part of BCT in early breast cancer patients [23].

Immediate tumor bed reconstruction used in this series is not routinely used for BCT. This approach allows for good cosmetics even in cases with large excision volume. Immediate catheter placement reduces the target volume; however, its determination remains subjective. In our series, the mean target volume was relatively small (34 cc). This may allow for better cosmetic outcome but at the expense of potentially increased risk of local recurrence. Harms et al. [5], in a series of patients with high risk of recurrence, reported the mean PDR boost volume of $57 \mathrm{cc}$. In the study by Resch et al. [24], in which $60 \%$ of patients underwent quadrantectomy, $18 \%$ wide excision and $22 \%$ tumorectomy, the average volume for the "prescribed dose" was $83 \mathrm{cc}$. Of note, in that study, the type of surgery did not impact the local control. Notably, excellent local control $(1.6 \%$ "true local recurrence" at 10 years for patients after quadrantectomy, $0 \%$ for wide excision, and $2.2 \%$ for tumorectomy) was accompanied by a fair cosmetic effect (excellent or good cosmetic effect in only $38 \%$ of patients).

In this series, three patients underwent surgical intervention for fat necrosis. In one of them, the treated volume was relatively high $\left(\mathrm{V}_{100}\right.$ of $\left.56.4 \mathrm{cc}\right)$. The DHI was available for two patients, and in both cases, it was 0.68 $\left(\mathrm{V}_{150}\right.$ of $10.7 \mathrm{cc}$ and $\left.17.8 \mathrm{cc}\right)$. The reported incidence of fat necrosis including symptomatic/clinically overt cases varies considerably in particular studies, and is influenced by differences in patient characteristics, treatment, duration of follow-up, and diagnostic criteria for a diagnosis of fat necrosis. No late fat necrosis was reported in the two above mentioned studies applying PDR BT boost at a dose of 15-25 Gy following whole breast irradiation $[4,5]$. The median follow-up in these series was 30 months and 60.9 months. In another small series with patients boosted with PDR BT with the median dose of 12.3 Gy (range: 12.0-20.3 Gy) (median $\mathrm{V}_{100}$ of $55.2 \mathrm{cc}$, median DHI: 0.82), in addition to 50.4 Gy WBRT, the incidence of mammographically evident signs of fat necrosis was $9.0 \%$ at the median follow-up of 37.5 months, but no patient needed surgical intervention [25]. After a median follow-up of 46 months, fat necrosis in one case $(1.3 \%)$ was observed in a series with HDR-BT (median $\mathrm{V}_{100}$ of $94.49 \mathrm{cc}$ ) performed immediately after completing WBRT [26]. The late toxicity data in patients treated with multicatheter interstitial HDR-BT as a form of accelerated partial breast irradiation (APBI), suggest that $\mathrm{V}_{150}$ and $\mathrm{V}_{200}$, as well as anthracycline-based chemotherapy administered after APBI may be associated with an increased risk of fat necrosis [27]. In another APBI study, acute breast infection and anthracycline-based chemotherapy, number of catheters, $\mathrm{V}_{100}, \mathrm{~V}_{150}, \mathrm{~V}_{200}$, and integrated reference air-kerma were associated with fat necrosis [28]. Of these, $V_{150}$ was independent treatment-related parameter. In these studies, the mean $\mathrm{V}_{100}$ was $176 \mathrm{cc}$ and $239 \mathrm{cc}$, respectively.

Our series includes 17 breast cancer patients (17.7\%) who were administered preoperative chemotherapy, an increasingly used strategy [29]. In this group, one patient developed angiosarcoma of the irradiated breast six years after BCT. Secondary angiosarcoma may develop in a lymphedematous arm, in the irradiated chest wall after radical mastectomy, and following BCT. This event is rare but associated with poor prognosis. In a large retrospective series of 18,115 breast cancers treated with BCT, including $50 \mathrm{~Gy}$ whole breast irradiation and a boost dose of 15-25 Gy, post-irradiation angiosarcoma was diagnosed in only nine cases, after the median latency period of approximately 74 months [30]. Adjuvant radiotherapy, an indispensable part of $\mathrm{BCT}$, is associated with the risk of second malignancy including all sarcomas and angiosarcoma [31]. Beside radiotherapy, partial mastectomies and lymph node dissections were found to be independent risk factors for the development of angiosarcoma in breast cancer patients [32]. Post-irradiation breast angiosarcoma, the most frequent second type of sarcoma after primary breast cancer, has been paradoxically increasingly reported since currently most women with breast cancer have long-term survival. In addition, the increasing use of intensity modulated radiation therapy (IMRT) and volumetric modulated arc therapy (VMAT), might be associated with a higher risk of mutagenesis. Due to the higher number of fields and monitor units, these newer techniques have been shown to have greater out-of-beam doses including higher low dose exposure of the normal structures. Whether angiosarcoma is induced by radiation or persistent edema, or has a multifactorial origin is not clear. Our patient with ypT2N1 breast cancer underwent axillary dissection and received irradiation at a standard dose of 50 Gy in 25 fractions with tangential fields to the breast and the supraclavicular region. Thus, despite the lack of clinical lymphedema, she might have had some minimal subclinical lymph stasis involving the breast. The addition of neoadjuvant chemotherapy consisting of 6 cycles of doxorubicin and docetaxel, might have also contributed to secondary malignancy in this patient.

\section{Conclusions}

Peri-operative PDR-BT with tube implantation at the time of surgery seems to be a safe and convenient boost method allowing for good local control and satisfactory cosmetic effect. The direct visualization of the operative site during surgery allows for precise defining of the tumor bed and decreases the risk of "geographical miss" in determining the target volume. However, in a proportion of patients, the treatment plan should be verified after the final histology is obtained. Therefore, this strategy necessitates careful patient selection and primary histological diagnosis.

\section{Acknowledgments}

Part of the data was presented at the $5^{\text {th }}$ Polish Brachytherapy Society Congress, June 2-4, 2016, Poznań, Poland.

\section{Disclosure}

Authors report no conflict of interest. 


\section{References}

1. Bartelink H, Horiot JC, Poortmans P et al. European Organization for Research and Treatment of Cancer Radiotherapy and Breast Cancer Groups. Recurrence rates after treatment of breast cancer with standard radiotherapy with or without additional radiation. N Engl J Med 2001; 345: 1378-1387.

2. Bartelink H, Maingon P, Poortmans $P$ et al. on behalf of the European Organization for Research and Treatment of Cancer Radiation Oncology and Breast Cancer Groups. Wholebreast irradiation with or without a boost for patients treated with breast-conserving surgery for early breast cancer: 20-year follow-up of a randomized phase 3 trial. Lancet Oncol 2015; 16: 47-56.

3. Frazier RC, Kestin LL, Kini V et al. Impact of boost technique on outcome in early-stage breast cancer patients. Am J Clin Oncol 2001; 24: 26-32.

4. Fritz P, Berns C, Anton HW et al. PDR brachytherapy with flexible implants for interstitial boost after breast-conserving surgery and external beam radiation therapy. Radiother Oncol 1997; 45: 23-32.

5. Harms W, Krempien R, Hensley FW et al. 5-year results of pulsed dose rate brachytherapy applied as a boost after breast-conserving therapy in patients at high risk for local recurrence from breast cancer. Strahlenther Onkol 2002; 178: 607-614.

6. Krishnan EC, Krishnan L, Cytaki EP et al. Radiobiological advantage of an immediate interstitial boost dose in conservative treatment of breast cancer. Int J Radiat Oncol Biol Phys 1990; 18: 419-424.

7. Manning MA, Arthur DW, Schmidt-Ullrich RK et al. Interstitial high-dose-rate brachytherapy boost: the feasibility and cosmetic outcome of a fractionated outpatient delivery scheme. Int J Radiat Oncol Biol Phys 2000; 48: 1301-1306.

8. Bedwinek J. Breast conserving surgery and irradiation: the importance of demarcating the excision cavity with surgical clips. Int J Radiat Oncol Biol Phys 1993; 26: 675-679.

9. DeBiose DA, Horwitz EM, Martinez AA et al. The use of ultrasonography in the localization of the lumpectomy cavity for interstitial brachytherapy of the breast. Int J Radiat Oncol Biol Phys 1997; 38: 755-759.

10. Regine WF, Ayyangar KM, Komarnicky LT et al. Computer-CT planning of the electron boost in definitive breast irradiation. Int J Radiat Oncol Biol Phys 1991; 20: 121-125.

11. Serkies K, Tarnawska Z, Blukis A et al. Comparison of two techniques of interstitial pulsed dose rate boost brachytherapy in conservative treatment of breast cancer. J Contemp Brachyther 2009; 1: 33-37.

12. Mansfield CM, Komarnicky LA, Schwartz GF et al. Perioperative implantation of iridium-192 as the boost technique for stage I and II breast cancer: results of a 10-year study of 655 patients. Radiology 1994; 192: 33-36.

13. Reitsamer R, Peintinger F, Sedlmayer F et al. Intraoperative radiotherapy given as a boost after breast-conserving surgery in breast cancer patients. Eur J Cancer 2002; 38: 1607-1610.

14. Vaidya JS, Baum M, Tobias JS et al. The novel technique of delivering targeted intraoperative radiotherapy (Targit) for early breast cancer. Eur J Surg Oncol 2002; 28: 447-454.

15. Veronesi U, Orecchia R, Luini A et al. A preliminary report of intraoperative radiotherapy (IORT) in limited-stage breast cancers that are conservatively treated. Eur J Cancer 2001; 37: 2178-2183.

16. Leong C, Boyages J, Jayasinghe UW et al. Effect of margins on ipsilateral breast tumor recurrence after breast conservation therapy for lymph node-negative breast carcinoma. Cancer 2004; 100: 1823-1832.

17. Sadek BT, Homayounfar G, Abi Raad RF et al. Is a higher boost dose of radiation necessary after breast-conserving therapy for patients with breast cancer with final close or positive margins? Breast Cancer Res Treat 2015; 154: 71-79.

18. Garvey EM, Senior DA, Pockaj BA et al. Rates of residual disease with close but negative margins in breast cancer surgery. Breast 2015; 24: 413-417.

19. Bosma SCJ, van der Leij F, van Werkhoven E et al. Very low local recurrence rates after breast-conserving therapy: analysis of 8485 patients treated over a 28 -year period. Breast Cancer Res Treat 2016; 156: 391-400.

20. Moran MS, Schnitt SJ, Giuliano AE et al. Society of Surgical Oncology-American Society for Radiation Oncology consensus guideline on margins for breast-conserving surgery with whole-breast irradiation in stage I and II invasive breast cancer. J Clin Oncol 2014; 32: 1507-1515.

21. Galimberti V, Taffurelli M, Leonardi MC et al. Surgical resection margins after breast-conserving surgery: Senonetwork recommendations. Tumori 2016; 102: 284-289.

22. Deore SM, Sarin R, Dinshaw KA et al. Influence of dose-rate and dose per fraction on clinical outcome of breast cancer treated by external beam irradiation plus iridium-192 implants: analysis of 289 cases. Int J Radiat Oncol Biol Phys 1993; 26: 601-606.

23. Budach W, Bolke E, Matuschek C. Hypofractionated radiotherapy as adjuvant treatment in early breast cancer. A review and meta-analysis of randomized controlled trials. Breast Care 2015; 10: 240-245.

24. Resch A, Potter R, Van Limbergen E et al. Long-term results (10 years) of intensive breast conserving therapy including a high-dose and large-volume interstitial brachytherapy boost (LDR/HDR) for T1/T2 breast cancer. Radiother Oncol 2002; 63: 47-58.

25. Ott OJ, Schulz-Wendtland R, Uter $W$ et al. Fat necrosis after conserving surgery and interstitial brachytherapy and/ or external-beam irradiation in women with breast cancer. Strahlenther Onkol 2005; 181: 638-644.

26. Gutiérrez C, Najjari D, Martinez E et al. The use of an interstitial boost in the conservative treatment of breast cancer: how to perform it routinely in a radiotherapy department. J Contemp Brachytherapy 2014; 6: 397-403.

27. Wazer DE, Kaufman S, Cuttino L et al. Accelerated partial breast irradiation: an analysis of variables associated with late toxicity and long-term cosmetic outcome after high-dose-rate interstitial brachytherapy. Int J Radiat Oncol Biol Phys 2006; 64: 489-495.

28. Garsa AA, Ferraro DJ, DeWees T et al. Analysis of fat necrosis following adjuvant high-dose-rate interstitial brachytherapy for early-stage breast cancer. Brachytherapy 2013; 12: 99-106.

29. Vugts G, Maaskant-Braat AJ, Niewenhuijzen GA et al. Patterns of care in the administration of neo-adjuvant chemotherapy for breast cancer. A population-based study. Breast J 2016; 22: 316-321.

30. Marchal C, Weber B, de Lafontan B et al. Nine breast angiosarcomas after conservative treatment for breast carcinoma: a survey from French Comprehensive Cancer Centers. Int J Radiat Biol Phys 1999; 44: 113-119.

31. Grantzau T, Overgaard J. Risk of second non-breast cancer after radiotherapy for breast cancer: a systematic review and meta-analysis of 762,468 patients. Radiother Oncol 2015; 114: 56-65.

32. Mery CM, George S, Bertagnolli MM et al. Secondary sarcomas after radiotherapy for breast cancer: sustained risk and poor survival. Cancer 2009; 115: 4055-4063. 